\title{
Influence of standardized activities on validity of Assessment of Capacity for Myoelectric Control
}

\author{
Helen Y. N. Lindner, MSc; ${ }^{1-2 *}$ Ann-Christin Eliasson, PhD; ${ }^{3}$ Liselotte M. N. Hermansson, PhD ${ }^{\mathbf{1 , 4}}$ \\ ${ }^{1}$ School of Health and Medical Sciences and ${ }^{2}$ Centre for Rehabilitation Research, Örebro University Hospital, Örebro, \\ Sweden; ${ }^{3}$ Department of Women's and Children's Health, Karolinska Institute, Stockholm, Sweden; ${ }^{4}$ Department of \\ Prosthetics and Orthotics, Örebro University Hospital, Örebro, Sweden
}

\begin{abstract}
The Assessment of Capacity for Myoelectric Control (ACMC) is an observation-based clinical tool that evaluates ability to control a myoelectric prosthetic hand during bimanual activities. Two validity aspects were investigated: potential bias interaction between prosthesis users and activities performed during assessment, and potential bias interaction between activities and different user characteristics (sex or prosthetic side). Six activities were standardized for the ACMC. Upper-limb myoelectric prosthesis users (47 congenital, 11 acquired; 31 male, 27 female, average age $19.9 \mathrm{yr}$ ) performed three standardized activities, each on one occasion. Bias-interaction analysis in the many-facet Rasch model identified inconsistent patterns in the interactions of individual users and activity facets and between activities and user characteristics. The standardized activities had no significant influence on measures of user ability. The activities functioned similarly across both sexes ( $p$-value greater than or equal to 0.12 ) and across both prosthetic sides in persons with upper-limb reduction deficiency ( $p$-value greater than or equal to 0.50$)$ and persons with acquired amputation ( $p$-value greater than or equal to 0.13 ). The results provide evidence for the validity of the ACMC across the standardized activities and support use of the ACMC in prosthesis users of both sexes and prosthetic sides. The newly standardized activities are recommended for future ACMC use.
\end{abstract}

Key words: activities, amputation, assessment, bimanual, instrument validation, myoelectric control, prostheses, rehabilitation, upper-limb reduction deficiency, upper limb.

\section{INTRODUCTION}

The Assessment of Capacity for Myoelectric Control (ACMC) is an observation-based clinical measure developed to assess an individual's ability to operate a myoelectric prosthetic hand [1-2]. The assessment focuses on how capably a prosthesis user operates a myoelectric prosthetic hand during bimanual activities [2]. Previous research on the evidence of validity for ACMC supports its unidimensionality; that is, all the items measure the same construct [3].

Instrument validation is a continual process, and one important aspect of validity that has not been previously evaluated in the ACMC is the degree to which the test scores are affected by processes that are extraneous to the construct, the so-called construct-irrelevant variance [4]. An ACMC assessment is typically carried out while the prosthesis user performs bimanual activities of his or her choice.

\footnotetext{
Abbreviations: $\mathrm{AA}=$ acquired amputation, $\mathrm{ACMC}=$ Assessment of Capacity for Myoelectric Control, LDAPC $=$ Limb Deficiency and Arm Prostheses Centre, MFRM = many-facet Rasch model, ULRD = upper-limb reduction deficiency.

*Address all correspondence to Helen Y. N. Lindner, PhD; Centre for Rehabilitation Research, Örebro University Hospital, SE 70185 Örebro, Sweden; +46-19-6025882, +4673-8048736; fax: +46-19-255559.

Email: helen.lindner@orebroll.se

http://dx.doi.org/10.1682/JRRD.2012.12.0231
} 
The activity, similar to the activities used in other studies in upper-limb prosthetics [5-8], is a tool applied to stimulate the prosthesis user to operate the prosthetic hand so that his or her ability can be assessed. The need for assistance during the activity and the activity result are not taken into consideration when scoring the user's ability. Hence, the ACMC is supposed to reflect only the user's ability to operate a myoelectric prosthetic hand. Nevertheless, it is uncertain whether the activities being used in the ACMC assessments have any influence on the ability measures. One way to evaluate this validity aspect is to examine whether prosthesis users would receive different ability measures when performing different activities. Thus, several bimanual activities need to be selected to standardize for the ACMC.

Another aspect of the activities addressed in this study is whether the new standardized activities function similarly between different sexes and prosthetic sides. In a previous validation of ACMC, it was shown that three items functioned slightly differently between male and female users [3]. As suggested in studies of other hand-skill instruments that use different activity choices, because men and women in general are familiar with different activities, the activities might favor a particular sex [9-10]. It is therefore important to examine this also in the ACMC. Furthermore, studies on the influence of prosthetic side among persons with acquired amputation (AA) have shown a difference in skill between users on the dominant versus nondominant side [11-13]. The question is, therefore, Do the activities function similarly across prosthetic sides? Further analysis of this question in a standardized situation will improve our knowledge of whether the differences among groups represent an activity problem or a true difference in the ability to operate a prosthetic hand.

Therefore, the aim of this study was to develop standardized activities and to examine the influence of these activities on the validity of the ACMC. This was achieved by investigating the following research questions: (1) Do prosthesis users receive different ability measures in the ACMC assessments for different standardized activities? (2) Do the standardized activities function similarly across different sexes and prosthetic sides?

\section{METHODS}

The study was carried out in two stages. Standardized activities were developed first and then used to evaluate the research questions.

\section{Development of Standardized Activities}

Certified ACMC raters from different countries (Canada, United States, Finland, Sweden, Norway, Netherlands, and Slovenia) were asked to suggest three activities that they would use in ACMC assessments. Some raters work with adult users $(n=25)$, and others work with pediatric users $(n=27)$. Overall, we received a total of 66 suggestions ( 9 activities were suggested by more than one rater). These suggestions were grouped into the following activity categories: self-care, household, and hobby/leisure (Table 1).

Considering the study aim and the feasibility of the data collection procedure, we decided to select and standardize six activities for this study. The selection of activities was based on four criteria: (1) all ACMC items must be easily

\section{Table 1.}

Certified raters' activity suggestions for Assessment of Capacity for Myoelectric Control (ACMC).

\begin{tabular}{|c|c|}
\hline Category & Activity Suggestion \\
\hline Self-Care & $\begin{array}{l}\text { Dressing. } \\
\text { Brushing teeth. } \\
\text { Eating and drinking. }\end{array}$ \\
\hline Household & $\begin{array}{l}\text { Changing car tires/car oil. } \\
\text { Installing a smoke alarm. } \\
\text { Grocery shopping/using wallet/stocking groceries } \\
\text { on shelves. } \\
\text { Making simple meals.* } \\
\text { Changing a bed. } \\
\text { Dishwashing. } \\
\text { Sorting mail. } \\
\text { Ironing. } \\
\text { Setting a table.* } \\
\text { Hanging laundry. } \\
\text { Washing small laundry items. } \\
\text { Setting up curtains. } \\
\text { Driving. } \\
\text { Packing a suitcase.* }\end{array}$ \\
\hline Hobby/Leisure & $\begin{array}{l}\text { Assembling a simple project (table, clipboard, } \\
\text { birdhouse, coat rack, LEGO bricks). } \\
\text { Painting. } \\
\text { Hanging pictures. } \\
\text { Sewing. } \\
\text { Fishing. } \\
\text { Repotting plants. } \\
\text { Pitching a tent. } \\
\text { Wrapping a gift. } \\
\text { Making handicrafts. } \\
\text { Playing doll dress-up. } \\
\text { Knitting. }\end{array}$ \\
\hline
\end{tabular}


observed, (2) the activities must be adaptable to suit different ages, (3) the activities must be easy to perform in a clinical setting, and (4) the time taken to complete the activity must be 10-15 min. Of the 66 suggestions, the following activities were chosen: "repotting a plant," "a ready-toassemble project” (e.g., build model with LEGO bricks, assemble a table lamp or an Advent star), "setting a table for four persons," "mixing a store-bought cake/pudding mix," "sorting bills or pictures," and "packing a suitcase for overnight stay.” Four ACMC raters from the United States, Canada, and Sweden discussed the standardization and documented the detailed steps for each activity. The activities were standardized according to objects, their placement in the room, and performance of the procedure. Materials for the activities were selected and suggested for different age groups and sexes, when necessary. Each activity was then pilot tested to ascertain that all 22 ACMC items could be observed in each activity and that the time taken to complete the activity stayed within the estimated $10-15 \mathrm{~min}$. For future use, all standardized activities will be documented in the ACMC training manual [2] and available for certified ACMC raters and future ACMC course participants.

\section{Participants}

Potential participants were recruited from the Limb Deficiency and Arm Prostheses Centre (LDAPC), Örebro University Hospital, Örebro, Sweden. The ACMC is intended for myoelectric prosthesis users of different characteristics, and therefore, the inclusion criteria were (1) unilateral (left or right) and bilateral prostheses users; (2) different prosthetic levels; (3) both sexes and different ages; (4) persons with upper-limb reduction deficiency (ULRD) and persons with AA; and (5) users with different years of prosthetic experience, that is, years fitted with myoelectric prosthesis. We planned to recruit 60 upperlimb prosthesis users. However, since one ACMC assesses one prosthetic hand, a bilateral user with two myoelectric hands was considered as two separate users and gave two ACMC assessments. Fifty-five unilateral prosthesis users and three bilateral prosthesis users (with five myoelectric prostheses and one passive prosthesis) fulfilled the inclusion criteria and were recruited between September 2009 and April 2011. Participants' demographic information was retrieved from their clinical records (Table 2). Information about the study was given twice to the prosthesis users: once sent to their homes and once when they arrived at the LDAPC for their regular visits. Formal written consent was obtained directly from the participants or,
Table 2.

Participant demographics.

\begin{tabular}{lcc}
\hline \multicolumn{1}{c}{ Variable } & $\begin{array}{c}\text { ULRD } \\
(\boldsymbol{n}=\mathbf{4 7})\end{array}$ & $\begin{array}{c}\text { AA } \\
(\boldsymbol{n}=\mathbf{~ 1 1})\end{array}$ \\
\hline Age, Median (range) & $9(2-39)$ & $42(25-72)$ \\
Sex & 21 & 10 \\
$\quad$ Male & 26 & 1 \\
$\quad$ Female & & \\
Side of Upper-Limb Loss & $14 / 33$ & $5 / 3$ \\
$\quad$ Unilateral (right/left; dominant/nondominant) & - & 3 \\
Bilateral & & \\
Level of Upper-Limb Loss & - & 3 \\
$\quad$ Unilateral-Transhumeral & 38 & 4 \\
Transradial & 9 & 1 \\
Carpal & - & 1 \\
Bilateral-Transhumeral and Transradial (2 myo) & - & 1 \\
Transradial (2 myo) & & 1 \\
Transhumeral (passive) and carpal (myo) & $0-36$ & $0-40$ \\
Years Fitted with Myo (range) & & \\
Prosthetic Wearing Time & 26 & 5 \\
Full Time (>8 h/d, 7 d/wk) & 7 & 3 \\
Part Time (4-8 h/d, 5-7 d/wk) & 8 & - \\
Occasional (<4 h, 1-7 d/wk) & 3 & 2 \\
Sporadic (at least once/mo) & 1 & - \\
Stopped wearing for 3 mo & 2 & 1 \\
None (new user) & & \\
\hline AA = acquired amputation, myo = myoelectric prostheses, ULRD & upper- \\
limb reduction deficiency. & & \\
\hline \hline
\end{tabular}

in the case of children, from their parents. Data collection took place at the LDAPC Occupational Therapy Unit. Ethical approval was obtained from the Uppsala Ethical Committee in Sweden.

\section{Outcome Measures}

The ACMC is an instrument designed to measure an individual's ability to control a myoelectric prosthetic hand during the performance of bimanual activities [2]. Internal scale validity and rater reliability have been demonstrated [1,3,14]. A recent validation of the ACMC [3] suggested elimination of one redundant item and combinations of items with similar levels of difficulty and therefore led to the current 22-item version. The items assess six aspects in relation to myoelectric control: need for external support for the prosthetic arm/hand, use of appropriate prosthetic grip force, ability to operate the hand in different positions/heights, ability to grasp and release objects repetitively, coordination of both hands, and need for visual feedback. All ACMC items are evaluated on a 4-point rating scale, ranging from 0 (not capable) to 3 (extremely capable). 
Prosthesis wearing time, that is, the number of hours the participant wore the prosthesis per day, was reported verbally by the participants or their parents. At the LDAPC, prosthetic wearing time is routinely categorized into five levels: 1 = full time, $>8 \mathrm{~h} / \mathrm{d}, 7 \mathrm{~d} / \mathrm{wk} ; 2=$ part time, 4-8 h/d, 5-7 d/wk; 3 = occasional, $<4$ h, 1-7 d/wk; $4=$ sporadic, at least once a month; and $5=$ nonuser, new user, or stopped wearing for a period.

\section{Procedures}

Participants were recruited during their regular clinical visit at the LDAPC. After the patients agreed to participate in the study, they were assigned to perform three of the six standardized activities. An allocation method called minimization [15] was used to assign activities to participants to ensure that each activity was performed by a similar group of person characteristics. This method ensures the balance between groups and is suggested in order to allow smaller trials than with those with typically performed randomization [16-18]. The user characteristics that were used for the minimization procedure were age, sex, prosthetic side (dominant vs nondominant), prosthetic level, and years fitted with myoelectric prosthesis. Because hand dominance is unknown in ULRD, right-sided absence was considered as dominant side for this group. The distribution of user characteristics for each activity is shown in Table 3.

The participants performed their three activities in random order. There was a 5-10 min break between each activity. In contrast to a regular ACMC assessment, all the activity performances were videotaped. The two bilateral participants with two myoelectric hands performed their three activities once; both hands were assessed using the same activity performance video but on separate occasions.

One rater (H. Y. N. Lindner) assessed all the activity videos to avoid the influence of rater severity from different raters. In addition, another rater (L. M. N. Hermansson) scored 10 videos of the first 10 participants and the results were discussed between the raters to confirm the scorings. The first rater scored all the videos for one activity before scoring another activity. This was to avoid giving similar ratings to the three ACMC assessments of the same prosthesis user. A total of 180 ACMC assessments, with 30 assessments for each activity, were collected.

\section{Data Analysis}

The 180 ACMC assessments were analyzed using the many-facet Rasch model (MFRM), an extension of the Rasch model derived by Linacre and Wright [19]. Detailed explanation of the MFRM is given elsewhere [19-20]. The MFRM allows the analysis of multiple aspects, or facets, and how they influence each other. When all the data were entered into the model, the MFRM calibrated three facets: user ability measure, item difficulty measure, and activity difficulty measure. All the measures were expressed in logits on a common linear scale with mean item difficulty set at 0 logits. A stable calibration of facets requires a minimum of 30 assessments per facet [21]. Each standardized activity had 30 assessments and therefore provided enough data for stable analyses.

Table 3.

Distribution of user characteristics across six standardized activities; figures represent number of people in each subgroup.

\begin{tabular}{|c|c|c|c|c|c|c|}
\hline \multirow[b]{2}{*}{ Activity } & \multirow[b]{2}{*}{ ULRD/AA } & \multicolumn{5}{|c|}{ Characteristics Used for "Minimization" Procedure ( $n=30$ /Activity) } \\
\hline & & $\begin{array}{c}\text { Sex } \\
\text { (male/female) }\end{array}$ & $\begin{array}{c}\text { Age } \\
(3-6 / 7-15 />15)\end{array}$ & $\begin{array}{l}\text { Prosthetic Side } \\
\text { (dominant/ } \\
\text { nondominant) }\end{array}$ & $\begin{array}{c}\text { Prosthetic Level } \\
\text { (transhumeral/ } \\
\text { transradial/carpal) }\end{array}$ & $\begin{array}{c}\text { Years Fitted with } \\
\text { Myoelectric } \\
\text { Prosthesis } \\
(0-4 / 5-10 />10)\end{array}$ \\
\hline Repotting Plant & $25 / 5$ & $16 / 14$ & $7 / 11 / 12$ & $10 / 20$ & $2 / 22 / 6$ & $5 / 7 / 18$ \\
\hline Ready-to-Assemble Project ${ }^{\dagger}$ & $23 / 7$ & $18 / 12$ & $4 / 13 / 13$ & $9 / 21$ & $2 / 22 / 6$ & $5 / 7 / 18$ \\
\hline Mixing Store-Bought Cake/Pudding Mix ${ }^{\dagger}$ & $25 / 5$ & $16 / 14$ & $5 / 13 / 12$ & $11 / 19$ & $2 / 22 / 6$ & $5 / 9 / 16$ \\
\hline Sorting Bills/Pictures ${ }^{\dagger}$ & $23 / 7$ & $16 / 14$ & $5 / 12 / 13$ & $13 / 17$ & $2 / 22 / 6$ & $5 / 9 / 16$ \\
\hline
\end{tabular}


The bias-interaction function in the MFRM was used to (1) investigate the activities in relation to the user ability and (2) investigate the sex and prosthetic side in relation to the activities. For the first question, the hypothesis was that prosthesis users would perform similarly in all three activities and hence give three similar ability measures. The bias size indicated whether, in this case, a user performed differently on any activity compared with the user mean ability measure. For the second question, a bias size was generated for the interaction between each activity and each user characteristic. To explore whether the hand dominance influenced the scores differently in different diagnostic groups, we performed separate analyses of persons with ULRD and persons with AA. The magnitude of the bias size indicated whether an activity was harder (-) or easier $(+)$ for any sex or prosthetic side compared with the activity difficulty measure based on the whole sample.

The MFRM also reported a $t$ statistic that tested the hypothesis that there was no significant interaction other than measurement error. As suggested, an interaction was considered significant when the magnitude of the bias size was greater than or equal to \pm 0.5 logits and the interaction was statistically significant $(p<0.05)$ [22]. The analyses were computed in FACETS computer program 3.70.2 (Winsteps; Chicago, Illinois). Independent sample $t$-test was used to test for other differences between groups.

\section{RESULTS}

\section{Participant Ability Measures}

Mean ability measure of the whole sample was 0.96 logits, with a higher mean in participants with ULRD than in participants with AA (Table 4). In both ULRD and AA, the mean ability measures of full-time prosthesis wearers were significantly higher (both $p=0.04$ ) than the mean ability measures of non-full-time wearers (Table 4). Further analysis showed that participants with dominantsided AA had higher ability measures than those with nondominant-sided AA ( $p=0.01)$, whereas no significant difference between sides was found in participants with ULRD.

\section{Influence of Standardized Activities on the User Ability Measures}

Table 5 shows the ability measures of the participants for their three assessments during three different
Table 4.

Prostheses users' Assessment of Capacity for Myoelectric Control (ACMC) ability measures among different user characteristics

\begin{tabular}{lcc}
\multicolumn{1}{c}{ Characteristic } & Persons with ULRD & Persons with AA \\
\hline Mean Ability (range) & $1.54(-4.63$ to 4.93$)$ & $-1.12(-3.37$ to 2.03$)$ \\
Full Time/Non-Full Time & $2.45 / 0.52$ & $-0.32 /-2.05$
\end{tabular}

(excluded new users)

\begin{tabular}{|c|c|c|c|c|}
\hline \multirow[b]{2}{*}{ Prosthetic Level } & Right & Left & Dominant & Nondominant \\
\hline & & & & \\
\hline Transhumeral & - & - & -1.36 & -3.34 \\
\hline Transradial & 1.89 & 1.40 & -0.46 & -1.26 \\
\hline Carpal & 1.66 & 1.43 & 2.03 & -2.57 \\
\hline
\end{tabular}

Note: ACMC ability measures are in logits. The higher the measure, the more able users are in subgroup.

${ }^{*}$ Right-sided ULRD is considered as dominant side.

AA = acquired amputation, ULRD = upper-limb reduction deficiency.

activities. Out of 60 prosthetic hands, 57 showed similar ability measures; that is, $<0.5$ logits difference between the activities. Two unilateral prosthesis users with ULRD and one bilateral prosthesis user with AA had $>0.5$ logits difference between their three ability measures, which was equivalent to 5 percent of the sample. Therefore, the ability measures of 95 percent of the sample were not significantly influenced by the standardized activities being performed during the assessments. When examining the three individuals in more detail, they all showed significant bias in the assessment of "packing a suitcase for overnight stay” (bias size 1.08, 0.78, and -0.99 logits, respectively). Two of them also showed significant bias in the assessment of "a ready-to-assemble project" (bias size -0.89 logits) and "repotting a plant" (bias size -1.43 logits). One user had experience with all three activities, whereas the other two reported that they had no experience with any of their three activities. No other pattern was found among these three participants, except that they were not full-time prosthesis users.

\section{Functioning of Standardized Activities Across Sex and Prosthetic Side}

The difficulty range of the six activities was between -0.67 and -1.08 logits (Table 6); that is, they were similar in terms of difficulty. Although bias-interaction analyses across sex and prosthetic side showed different bias sizes in most of the activities (Table 6), they were all within \pm 0.5 logits and did not differ significantly among males and females $(p \geq 0.17)$ or among dominant and nondominant prosthesis users (ULRD $p \geq 0.59$; AA $p \geq$ 0.33 ). This implies that the activities functioned similarly across both subgroups in terms of sex and prosthetic side. 
Table 5.

Prosthetic users’ Assessment of Capacity for Myoelectric Control ability measures in each of their three activities categorized according to prosthesis wearing time. Each line represents one prosthesis user (bilateral users with two myoelectric hands have two lines).

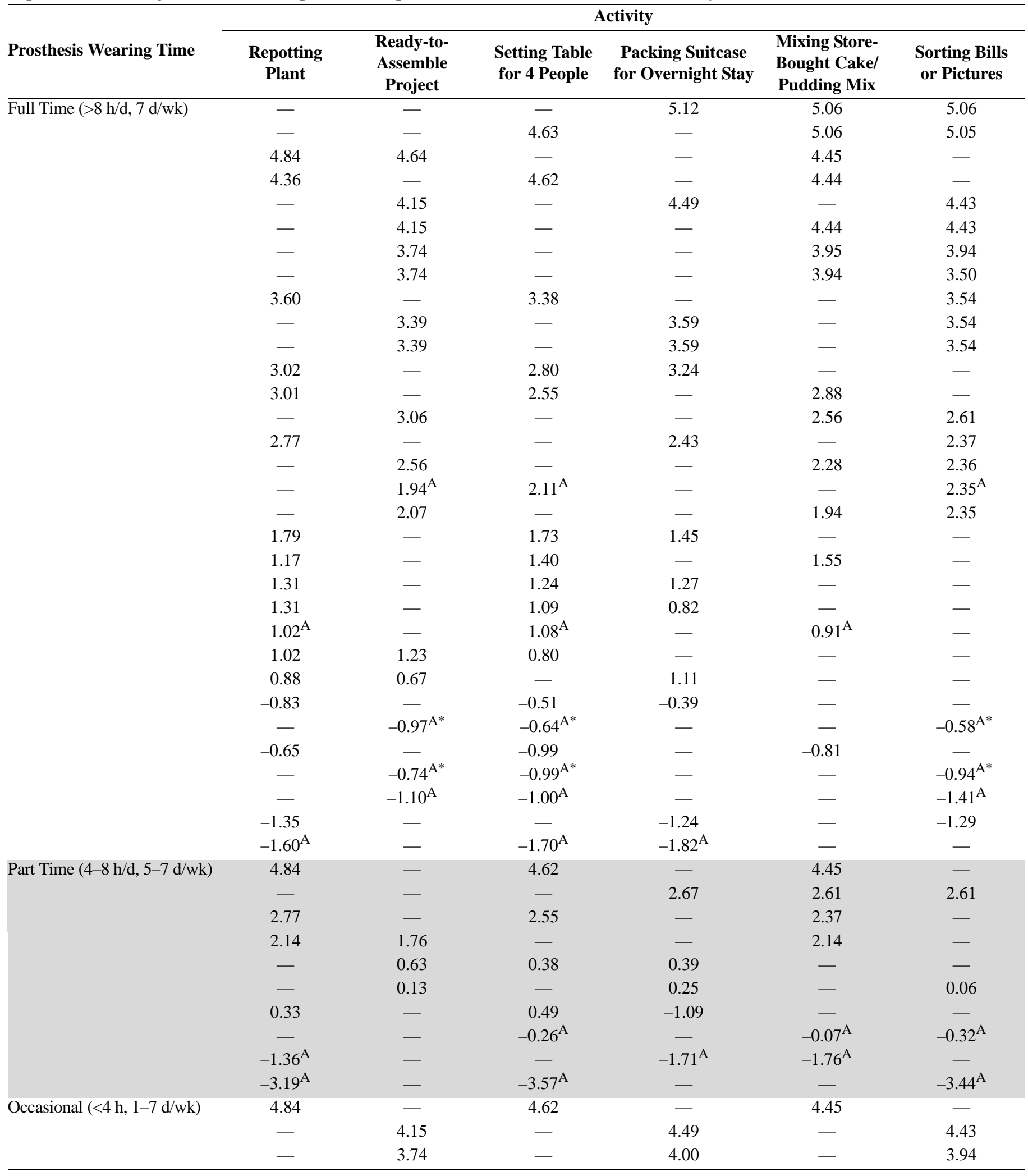


Table 5. (cont.)

Prosthetic users' Assessment of Capacity for Myoelectric Control ability measures in each of their three activities categorized according to prosthesis wearing time. Each line represents one prosthesis user (bilateral users with two myoelectric hands have two lines).

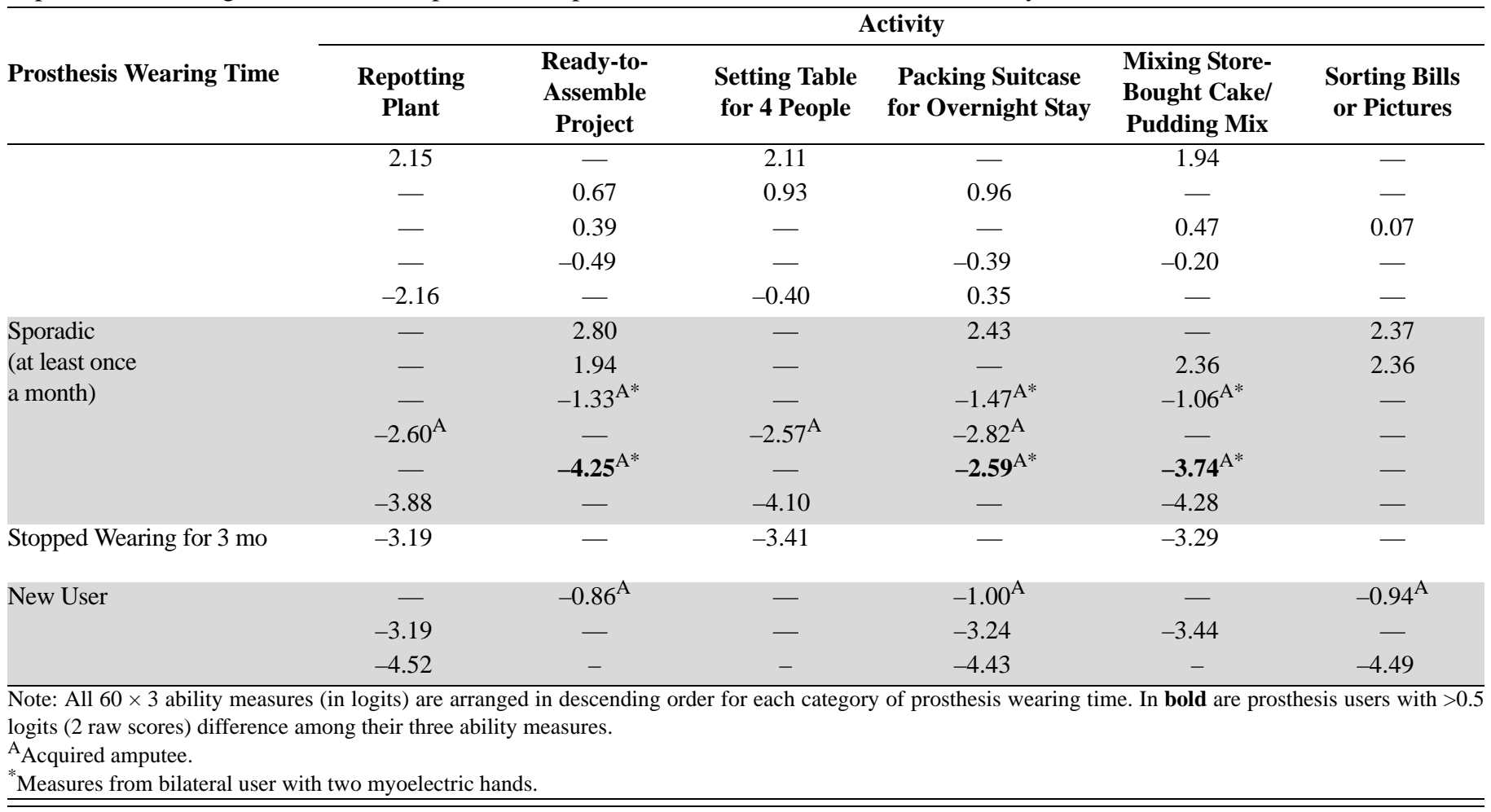

Table 6.

Activity difficulty, bias size, and $t$ statistics of interactions between activity, sex, and prosthetic side on Assessment of Capacity for Myoelectric Control ability measures.

\begin{tabular}{|c|c|c|c|c|c|c|c|c|c|c|c|c|c|}
\hline \multirow{3}{*}{ Activity } & \multirow{3}{*}{$\begin{array}{l}\text { Activity } \\
\text { Difficulty } \\
\text { Measure }\end{array}$} & \multicolumn{4}{|c|}{ Sex } & \multicolumn{4}{|c|}{ Persons with ULRD } & \multicolumn{4}{|c|}{ Persons with AA } \\
\hline & & \multicolumn{2}{|c|}{ Male } & \multicolumn{2}{|c|}{ Female } & \multicolumn{2}{|c|}{ Right $^{*}$} & \multicolumn{2}{|c|}{ Left } & \multicolumn{2}{|c|}{ Dominant } & \multicolumn{2}{|c|}{ Nondominant } \\
\hline & & $\overline{\text { Bias Size }}$ & $p$-Value & $\overline{\text { Bias Size }}$ & $p$-Value & Bias Size & $p$-Value & Bias Size & $p$-Value & Bias Size & $p$-Value & $\overline{\text { Bias Size }}$ & $p$-Value \\
\hline Repotting & -0.67 & +0.04 & 0.17 & -0.03 & 0.33 & 0.00 & 0.99 & -0.02 & 0.86 & -0.13 & 0.61 & -0.04 & 0.86 \\
\hline Setting Table & -0.90 & +0.01 & 0.74 & +0.01 & 0.81 & -0.04 & 0.84 & -0.05 & 0.65 & +0.08 & 0.63 & +0.09 & 0.60 \\
\hline Packing & -1.02 & -0.02 & 0.62 & +0.03 & 0.24 & -0.06 & 0.71 & -0.06 & 0.59 & +0.14 & 0.49 & -0.10 & 0.64 \\
\hline Mixing & -1.08 & -0.01 & 0.74 & +0.02 & 0.43 & +0.02 & 0.92 & -0.06 & 0.60 & -0.03 & 0.87 & +0.36 & 0.38 \\
\hline
\end{tabular}

Note: Activity difficulty measure and bias size are in logits. The higher the difficulty measure, the more difficult it is. Magnitude of bias size indicates whether activity is harder (-) or easier (+) for user characteristic subgroup compared with overall activity difficulty measure. $p$-Value is for $t$ statistic that tested hypothesis that no user characteristic-activity interaction exists.

*Right-sided upper-limb reduction deficiency (ULRD) is considered as dominant side.

\section{DISCUSSION}

In this study, we standardized six activities for the ACMC and determined that the user ability measures were not influenced by these standardized activities. Furthermore, all these activities functioned similarly across both prosthetic sides and both sexes.
Upper-limb prosthetic outcome measures often assess prosthetic skill during activity performance. Some of these measures have a standard set of activities or tasks [23-24], whereas others have different activities for different age groups [25-26] or client-chosen activities [1]. Until now, those measures that have different activities [1,25-26] have not been investigated with regard to whether the 
activities used during the assessments have any influence on the assessment results. To evaluate the possible influence of the activity on the assessment results, we standardized six activities for the ACMC. The result from the present study showed that the majority (57 out of 60; 95\%) of the prosthesis users scored similar ability measures on their three standardized ACMC activities. This supports the assumption that the standardized activities are extraneous to the ACMC construct and suggests that the influence of the activities on the ability measures is minimal. Hence, the users' ability measures are comparable when these activities are used.

An important aspect of any outcome measure is that it functions similarly across different participants, allowing for the direct comparison of data from different groups of people. This is especially important for the small population of upper-limb prosthesis users, for whom combined data from different centers or countries are often needed to make statistical comparisons. We expected the standardized activities to function differently based on user characteristics because this was reported in studies using other performance tests. There have been significant differences between males and females [9] and between dominant and nondominant sides [27-28]. However, none of the standardized activities in our study favored a particular prosthetic side or sex. Furthermore, the results were similar in both diagnostic groups. This suggests that ACMC data from people with these two diagnoses and user characteristics can be pooled and compared.

A potential difference between the two diagnostic groups in the sample was explored through separate analyses of the two groups. The only difference that was seen was a higher mean ability in ULRD than AA and this was probably due to the sample; there were relatively fewer full-time wearers in the AA group (50\%) than the ULRD group (58\%). One similarity in both AA and ULRD groups was that full-time prosthesis wearers had higher ability measures than non-full-time wearers. This is in accordance with the findings of a previous study in which functionality among children with ULRD was evaluated [29]. Adaptation to the prosthesis often encourages the users to increase their wearing time [29-30]. This may stimulate the clients to use their prostheses in daily activities and hence achieve better myoelectric control, which can explain the difference related to wearing pattern. Furthermore, similar to a finding among body-powered prosthesis users [31], significantly higher ability measures were found among persons with dominant-sided AA. The difference in these results between the diagnostic groups may be related to the fact that in AA, the handedness is established before amputation occurs. In contrast, in ULRD the handedness is established on the nondeficient side during growth and the effect of handedness on prosthetic ability is smaller than for people with AA at later age. Despite this unclear information, the result suggests that innate dominance may contribute to better myoelectric control. Further studies with large samples in both diagnostic groups are needed to confirm these findings.

\section{Strengths and Limitations}

The rigor of the study protocol and the use of the minimization method to reduce bias from extraneous factors added strength to this study. Also, the use of one rater removed the potentially negative influence of several raters in the analysis. However, the use of one rater added a potential systematic error to the ratings and thus reduced the reliability of the findings. We tried to overcome this problem by comparing a subset of ratings with initial ratings made by another author on the same videos. The ratings that differed between the authors were discussed until an agreement was reached. These agreed-upon definitions were then used for all future ratings. The difference is probably due to a difference in the raters' understandings of the items. Interrater reliability had earlier been demonstrated [14], but it is never perfect.

The participant sample was a potential limitation to the study. According to the study design, the prosthesis users were self-selected. Although the distribution of prosthetic skill among participants was good, there was a relatively small number of less-capable users (people with ability measures lower than the mean) in the sample (Table 5). The three users with bias interaction on the standardized activities were all less-capable users; therefore, the sample may have influenced the results in a positive way and thus a sample with an increased number of lesscapable users is needed to confirm our findings. Furthermore, the bias analysis on both sexes and prosthetic sides could be strengthened by a larger cohort.

\section{Study Implications}

Despite the study limitations, this study added meaningful evidence to the ACMC. One major clinical implication is the evidence for use of any of the six standardized activities for the assessment. In addition, the user ability measures are comparable when different standardized activities are used in the assessments. Another important implication is that by 
using the standardized activities, the scoring of all items may become easier for the raters and hence no item will be left as missing.

\section{CONCLUSIONS}

The ACMC is valid across the standardized activities and can be used to evaluate the capacity for myoelectric control in prosthesis users with different sexes and prosthetic sides. The newly standardized activities are recommended for future use of the ACMC.

\section{ACKNOWLEDGMENTS}

\section{Author Contributions:}

Study concept and design: H. Y. Lindner, A.-C. Eliasson,

L. M. Hermansson.

Acquisition of data: H. Y. Lindner.

Analysis and interpretation of data: H. Y. Lindner.

Drafting of manuscript: H. Y. Lindner, L. M. Hermansson.

Critical revision of manuscript for important intellectual content:

A.-C. Eliasson, L. M. Hermansson.

Statistical analysis: H. Y. Lindner.

Study supervision: A.-C. Eliasson, L. M. Hermansson.

Financial Disclosures: The authors have declared that no competing interests exist.

Funding/Support: This material was based on work supported by a doctoral grant from Health Care Sciences Postgraduate School, Karolinska Institute, Solna, Sweden, and a research grant from the Research Committee of Örebro County Council, Örebro, Sweden.

Additional Contributions: We would like to express our gratitude to Wendy Hill, research occupational therapist at the Institute of Biomedical Engineering, University of New Brunswick, Canada, and Kathy Stubblefield, research occupational therapist at the Neural Engineering Center for Artificial Limbs, Rehabilitation Institute of Chicago, Illinois, for their valuable advice on the development of standardized activities. A special thanks to the upper-limb prosthesis users from LDAPC who gave their time for this study. Helen Y. N. Lindner received her $\mathrm{PhD}$ in October 2013.

Institutional Review: The study was approved by the Uppsala Ethical Committee in Sweden (No. 231). Formal written consent was obtained directly from the participants or, in the case of children, from their parents.

Participant Follow-Up: The study abstract in Swedish will be published on the Örebro University Hospital Web site.

\section{REFERENCES}

1. Hermansson LM, Fisher AG, Bernspång B, Eliasson AC. Assessment of capacity for myoelectric control: A new
Rasch-built measure of prosthetic hand control. J Rehabil Med. 2005;37(3):166-71. [PMID:16040474] http://dx.doi.org/10.1080/16501970410024280

2. Hermansson LM, Hill W, Lindner HY. Assessment of Capacity for Myoelectric Control version 2.0 training manual. Örebro (Sweden): Örebro University Hospital; 2011.

3. Lindner HY, Linacre JM, Norling Hermansson LM. Assessment of capacity for myoelectric control: Evaluation of construct and rating scale. J Rehabil Med. 2009;41(6): 467-74. [PMID:19479160] http://dx.doi.org/10.2340/16501977-0361

4. American Educational Research Association, American Psychological Association, National Council on Measurement in Education. Standards for educational and psychological testing. Washington (DC): AERA Publications; 1999.

5. Bouwsema H, van der Sluis CK, Bongers RM. The role of order of practice in learning to handle an upper-limb prosthesis. Arch Phys Med Rehabil. 2008;89(9):1759-64. [PMID:18675393] http://dx.doi.org/10.1016/j.apmr.2007.12.046

6. Bouwsema H, van der Sluis CK, Bongers RM. Learning to control opening and closing a myoelectric hand. Arch Phys Med Rehabil. 2010;91(9):1442-46. [PMID:20801265] http://dx.doi.org/10.1016/j.apmr.2010.06.025

7. Stubblefield KA, Miller LA, Lipschutz RD, Kuiken TA. Occupational therapy protocol for amputees with targeted muscle reinnervation. J Rehabil Res Dev. 2009;46(4):481-88. [PMID:19882483] http://dx.doi.org/10.1682/JRRD.2008.10.0138

8. Weeks DL, Wallace SA, Anderson DI. Training with an upper-limb prosthetic simulator to enhance transfer of skill across limbs. Arch Phys Med Rehabil. 2003;84(3):437-43. [PMID:12638114] http://dx.doi.org/10.1053/apmr.2003.50014

9. Chien CW, Brown T, McDonald R. Rasch analysis of the assessment of children's hand skills in children with and without disabilities. Res Dev Disabil. 2011;32(1):253-61. [PMID:21041063] http://dx.doi.org/10.1016/j.ridd.2010.09.022

10. Merritt BK, Fisher AG. Gender differences in the performance of activities of daily living. Arch Phys Med Rehabil. 2003;84(12):1872-77. [PMID:14669197] http://dx.doi.org/10.1016/S0003-9993(03)00483-0

11. Burger H, Franchignoni F, Heinemann AW, Kotnik S, Giordano A. Validation of the orthotics and prosthetics user survey upper extremity functional status module in people with unilateral upper limb amputation. J Rehabil Med. 2008; 40(5):393-99. [PMID:18461266] http://dx.doi.org/10.2340/16501977-0183

12. Gaine WJ, Smart C, Bransby-Zachary M. Upper limb traumatic amputees. Review of prosthetic use. J Hand Surg [Br]. 1997;22(1):73-76. [PMID:9061532] http://dx.doi.org/10.1016/S0266-7681(97)80023-X 
13. Jang CH, Yang HS, Yang HE, Lee SY, Kwon JW, Yun BD, Choi JY, Kim SN, Jeong HW. A survey on activities of daily living and occupations of upper extremity amputees. Ann Rehabil Med. 2011;35(6):907-21. [PMID:22506221] http://dx.doi.org/10.5535/arm.2011.35.6.907

14. Hermansson LM, Bodin L, Eliasson AC. Intra- and inter-rater reliability of the assessment of capacity for myoelectric control. J Rehabil Med. 2006;38(2):118-23. [PMID:16546769] http://dx.doi.org/10.1080/16501970500312222

15. Altman D. Practical statistics for medical research. London (UK): Chapman \& Hall/CRC; 1999.

16. Altman DG, Bland JM. Treatment allocation by minimisation. BMJ. 2005;330(7495):843.

17. Scott NW, McPherson GC, Ramsay CR, Campbell MK. The method of minimization for allocation to clinical trials. a review. Control Clin Trials. 2002;23(6):662-74. [PMID:12505244] http://dx.doi.org/10.1016/S0197-2456(02)00242-8

18. Taves DR. The use of minimization in clinical trials. Contemp Clin Trials. 2010;31(2):180-84. [PMID:20060500] http://dx.doi.org/10.1016/j.cct.2009.12.005

19. Linacre JM, Wright BD. Construction of measures from many-facet data. J Appl Meas. 2002;3(4):484-512.

20. Eckes T. Introduction to many-facet Rasch measurement: Analyzing and evaluating rater-mediated assessments: New York (NY): Peter Lang Publishing Group; 2011.

21. Linacre JM. A user's guide to FACETs-Rasch-model computer programs. [place unknown]: John M. Linacre; 2012.

22. Tristan A. An adjustment for sample size in DIF analysis. Rasch Measure Trans. 2006;20(3):1070-71.

23. Light CM, Chappell PH, Kyberd PJ. Establishing a standardized clinical assessment tool of pathologic and prosthetic hand function: Normative data, reliability, and validity. Arch Phys Med Rehabil. 2002;83(6):776-83. [PMID:12048655] http://dx.doi.org/10.1053/apmr.2002.32737

24. Resnik L, Adams L, Borgia M, Delikat J, Disla R, Ebner C, Walters LS. Development and evaluation of the Activities Measure for Upper Limb Amputees. Arch Phys Med Rehabil. 2013;94(3):488-94.e4.

25. Bagley AM, Molitor F, Wagner LV, Tomhave W, James MA. The Unilateral Below Elbow Test: A function test for children with unilateral congenital below elbow deficiency. Dev Med Child Neurol. 2006;48(7):569-75. [PMID:16780626] http://dx.doi.org/10.1017/S0012162206001204
26. Sanderson E, Scott R. UNB test of prosthetic function: a test for unilateral amputees (test manual). Fredericton (Canada): University of New Brunswick; 1985.

27. Bryden PJ, Roy EA. A new method of administering the Grooved Pegboard Test: Performance as a function of handedness and sex. Brain Cogn. 2005;58(3):258-68. [PMID:15963376] http://dx.doi.org/10.1016/j.bandc.2004.12.004

28. Gordon HW, Kravetz S. The influence of gender, handedness, and performance level on specialized cognitive functioning. Brain Cogn. 1991;15(1):37-61. [PMID:2009173] http://dx.doi.org/10.1016/0278-2626(91)90014-Y

29. Korkmaz M, Erbahçeci F, Ulger O, Topuz S. Evaluation of functionality in acquired and congenital upper extremity child amputees. Acta Orthop Traumatol Turc. 2012;46(4): 262-68. [PMID:22951757] http://dx.doi.org/10.3944/AOTT.2012.2631

30. Huizing K, Reinders-Messelink H, Maathuis C, HaddersAlgra M, van der Sluis CK. Age at first prosthetic fitting and later functional outcome in children and young adults with unilateral congenital below-elbow deficiency: A crosssectional study. Prosthet Orthot Int. 2010;34(2):166-74.

[PMID:20298129]

http://dx.doi.org/10.3109/03093640903584993

31. Dudkiewicz I, Gabrielov R, Seiv-Ner I, Zelig G, Heim M. Evaluation of prosthetic usage in upper limb amputees. Disabil Rehabil. 2004;26(1):60-63. [PMID:14660200] http://dx.doi.org/10.1080/09638280410001645094

Submitted for publication December 20, 2012. Accepted in revised form June 10, 2013.

This article and any supplementary material should be cited as follows:

Lindner HY, Eliasson AC, Hermansson LM. Influence of standardized activities on validity of Assessment of Capacity for Myoelectric Control. J Rehabil Res Dev. 2013; 50(10):1391-1400.

http://dx.doi.org/10.1682/JRRD.2012.12.0231

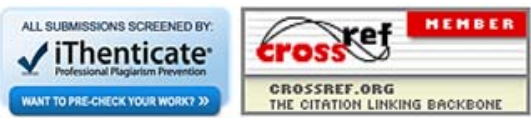

